\title{
Dialog und Theologie der Religionen
}

\author{
Reinhold Bernhardt
}

\section{Dokumentationen / Textsammlungen}

Ulrich Dehn u.A. (Hg.), Handbuch Dialog der Religionen. Christliche Quellen zur Religionstheologie und zum interreligiösen Dialog. Verlag Otto Lembeck, Frankfurt a.M. 2008, 472 S. - ERnst Fürlinger (Hg.), »Der Dialog muss weitergehen«. Ausgewählte vatikanische Dokumente zum interreligiösen Dialog (1964-2008). Herder, Freiburg/Basel/Wien 2009, 591 S. - CIBEDO e.V. (Hg.), Die offiziellen Dokumente der katholischen Kirche zum Dialog mit dem Islam, zusammengestellt von Tıмо Güzelmansur. Pustet, Regensburg 2009, 621 S. - Wolfram Weiße (Hg.), Theologie im Plural. Eine akademische Herausforderung (Religionen im Dialog 1). Waxmann, Münster u.a. 2009, 214 S. - Sonja Haug / Stephanie Müssig / Anja Stichs (im Auftrag der Deutschen Islam-Konferenz.), Muslimisches Leben in Deutschland (Forschungsbericht 6). Bundesamt für Migration und Flüchtlinge, Nürnberg 2009, 448 S. Deutsche Islam Konferenz, Drei Jahre Deutsche Islam Konferenz (DIK) 2006-2009. Muslime in Deutschland - deutsche Muslime. Bundesministerium des Innern, Berlin 2009, 419 S.

Je länger die Bemühungen um eine dialogische Gestaltung der interreligiösen Beziehung währen und je intensiver sie vorangetrieben werden, umso mehr Erfahrungen werden damit gesammelt, reflektiert und dokumentiert. Es entstehen Praxisberichte, Projektvorstellungen und Textsammlungen für pädagogische Zwecke. Im ersten Teil dieser Sammelbesprechung zu Neuerscheinungen aus dem Gebiet des Religionsdialogs und der Religionstheologie sollen einige dieser Dokumentationen bzw. Textsammlungen vorgestellt werden.

Einen ausgezeichneten Überblick über die im 20. Jh. geführte theologische Debatte zur Beziehungsbestimmung und -gestaltung zwischen Christentum und anderen Religionen gibt das von Ulrich DEHN hg. »Handbuch Dialog der Religionen«. Neben klassischen Texten deutschsprachiger (Troeltsch, Barth, Rahner, Pannenberg und Tillich) und niederländischer (Kraemer) Theologen wurden auch wichtige Beiträge asiatischer (Panikkar, Pieris, D'Sa) und anglo-amerikanischer Autoren (Hick, Swidler und Knitter) aufgenommen. Mit Georges Khodre ist ein prominenter Repräsentant der orthodoxen Theologie vertreten. Hans Jochen Margulls wegweisende »Bemerkungen zum Dialog« aus dem Jahr 1974 sind ebenso enthalten wie Hans Küngs pro- 
grammatische Überlegungen zum »Projekt Weltethos« und Perry SchmidtLeukels Versuch, die Einteilung religionstheologischer Standpunkte (Exklusivismus, Inklusivismus und Pluralismus) als logisch umfassende und notwendige Klassifikation zu erweisen. Im zweiten Teil des Readers sind kirchliche Dokumente zusammengestellt: Auszüge aus Erklärungen des Zweiten Vatikanischen Konzils (»Lumen Gentium« und »Nostra Aetate«), die maßgeblichen Verlautbarungen des ÖRK-Dialogprogramms (»Leitlinien zum Dialog mit Menschen verschiedener Religion und Ideologien« aus dem Jahre 1979 und die »Ökumenischen Erwägungen zum Dialog und zu den Beziehungen mit Menschen anderer Religionen« von 2002) sowie die »Theologische(n) Leitlinien« der EKD: „Christlicher Glaube und nichtchristliche Religionen« (2003).

Der Band ist mit einer instruktiven Einleitung des Herausgebers versehen, den einzelnen Texten sind kurze Einführungen vorangestellt. Diese Quellensammlung stellt eine wertvolle Materialgrundlage für Lehrveranstaltungen zu diesem Themenkomplex dar, eignet sich aber auch für die individuelle Lektüre. Ihre selbstgesetzte Grenze liegt in der weitgehenden Ausblendung der aktuellen religionstheologischen Debatte. Beiträge von zeitübergreifender Bedeutung aus dieser schnell voranschreitenden Diskussion ${ }^{1}$ herauszuheben, erscheint kaum möglich. Erst im Rückblick auf den geronnenen Fluss kristallisieren sich die Positionen heraus, die seinen Verlauf bestimmt haben.

Die Beiträge bewegen sich zumeist auf der (Meta-) Ebene des >Dialogs über den Dialog‘. Sie lassen sich vier thematischen Bereichen zuordnen:

(a) Einige fragen nach Regeln für die interreligiöse und interkulturelle Kommunikation und entwickeln eine sEthik des Dialogss. Vorstellungen von herrschaftsfreien Beziehungsgestaltungen werden dabei auf die Besonderheiten der interkulturellen und -religiösen Kommunikation übertragen. Es stellen sich aber auch Fragen: »Religionen《 sind keine Subjekte, die miteinander in Beziehung treten können. Insofern ist das Konzept eines »Dialogs der Religionen « in die abendländische Sicht von Religionen als nach innen relativ homogenen und nach außen relativ klar abgrenzbaren Einheiten eingebunden. Die Fragwürdigkeit dieser Sicht ist in den letzten Jahrzehnten auch von der westlichen Religionswissenschaft (etwa Wilfred Cantwell Smith), Religionssoziologie (etwa: Ulrich Beck) und Theologie (etwa in der Rezeption der sog. postcolonial studies) erkannt worden. Diese Kritik an einer pauschalisierenden Verwendung des Begriffs »Dialog der Religionen« führt zum einen dazu, die sich begegnenden religiösen Individuen als wirkliche Subjekte der Kommunikation in den Blick zu nehmen und damit auch zwischen verschiedenen interreligiösen Beziehungen genauer zu unterscheiden, und zum anderen den Kontext, in dem die Begegnung stattfindet, und

1 Siehe dazu R. Bernhardt, Literaturbericht »Theologie der Religionen«, ThR 72 (2007) 1-35.127-149. 
damit auch die Machtverteilung in der jeweiligen Kommunikationssituation stärker zu gewichten. Dadurch wird eine idealisierende Sicht des Dialoggeschehens verhindert.

(b) Andere Beiträge kreisen um die Frage einer sinterreligiösen Hermeneutikı: Wie ist das Verstehen einer anderen religiösen Tradition möglich? Lässt sich das Andere als Anderes überhaupt verstehen? Oder finden nicht doch immer wieder hermeneutische Integrations-, Inklusions- und Assimilationsprozesse statt, bei denen das >Andere im Kategorien- und Wertungsraster des `Eigenen` wahrgenommen wird? Die Antwort auf diese Frage ist von Bedeutung für die Entwicklung religionstheologischer Konzepte, besonders für die Kritik spluralistischer Ansätze der Religionstheologie, in denen die Vielfalt der Religionen auf ein letztes, ihnen allen transzendentes Einheitsprinzip bezogen wird. Damit geraten diese in die Gefahr, eine unerschwingliche Perspektive einzunehmen. Der postulierte Einheitsgrund bleibt entweder vollkommen unbestimmt oder wird im Licht der verschiedenen Traditionen verschieden bestimmt.

(c) Der größte Teil besonders der klassischen Texte setzt sich mit religionsphilosophischen und theologischen Grundfragen der Religionsvielfalt auseinander. Dabei geht es weniger um die konkrete Begegnung und mehr um die Deutung der Pluralität und der damit verbundenen konfligierenden Wahrheitsansprüche sowie um die Ortsbestimmung dieses Themas in der Theologie.

(d) Einige der Texte verlassen die metadialogische religionshermeneutische und -theologische Reflexionsebene und unternehmen interreligiöse Vergleiche. So fragt etwa Francis D'Sa nach dem Kern der christlichen Religion im Gegenüber zum indischen Dharma, um beide zueinander in Beziehung zu setzen. Der christlichen Religion liege die Erfahrung des Angenommenseins zugrunde, während die Hindu-Religiosität auf der Erfahrung des Getragenseins durch das All-Ganze ausgerichtet sei. Diese beiden Erfahrungen seien grundlegend verschieden, das Subjekt jedoch, das sich ihnen aussetze, sei das gleiche. In ihm könne eine gegenseitige Vermittlung dieser Grunderfahrungen und der Weltbilder, in die sie eingebunden sind, stattfinden: »Das kosmozentrische Weltbild muß das in ihm vorhandene Geschichtsbewusstsein und das anthropozentrische Weltbild das in ihm vorhandene mythische Bewusstsein entdecken lernen« (283).

Durch die Vielfalt der in ihm versammelten Perspektiven gibt der Band einen umfassenden Ein- und Überblick über die Vielschichtigkeit der Diskussion um die Beziehung des Christentums zu anderen Religionen. Während die evangelischen Kirchen nur relativ wenige Stellungnahmen zu diesem Thema hervorgebracht haben, gibt es auf römisch-katholischer Seite eine Fülle von lehramtlichen Äußerungen aus den Jahrzehnten seit dem Zweiten Vatikanischen Konzil. Sie füllen einen (vom »Päpstlichen Rat für den Interreligiösen Dialog« herausgegebenen) Band mit 1750 Seiten! ${ }^{2}$

Angeregt durch einen diesbezüglichen Wunsch, den Kardinal Lehmann 2002 in einem Grundsatzreferat vor der Deutschen Bischofskonferenz geäuBert hatte, hat ERnst Fürlinger die wichtigsten dieser Dokumente aus-

2 Pontifical Council for Interreligious Dialogue: Interreligious Dialogue. The Official Teaching of the Catholic Church. From the Second Vatican Council to John Paul II (1963-2005), hg. von Francesco Gioia, Boston: Pauline Books 2006. 
zugsweise in einem Band zusammengestellt. Ein programmatisches Wort von Johannes Paul II. gibt der Sammlung ihren Titel: »Der Dialog muss weitergehen«. In der Wahl dieses Titels drückt sich aus, dass F. nicht nur ein historisch-dokumentarisches Interesse verfolgt, sondern ein gegenwarts- und zukunftsbezogenes. In einer Zeit, in der religiös motivierte Spannungen und Konflikte anzuwachsen scheinen, sich eine islamkritische Stimmung aufbaut, vor Blauäugigkeit in der interreligiösen Begegnungspraxis gewarnt wird und die christlichen Kirchen bestrebt sind, das je eigene Profil interkonfessionell wie interreligiös zu schärfen, fordert er eine Fortsetzung und Intensivierung der Bemühungen um dialogische Beziehungsformen. Er kann sich dafür auf Lehmann berufen, der in seinem Vorwort zu diesem Band schreibt: „Vieles ist schon erreicht, der Weg muss aber weitergehen.«

Während die oben genannte umfassende Sammlung des »Päpstlichen Rates für den Interreligiösen Dialog« nur den Zeitraum bis 2005 abdeckt, sind in F.s Band Texte bis zum Jahr 2008 - und damit auch die Stellungnahmen von Benedikt XVI. - enthalten. Der Herausgeber hat sie mit kurzen, sehr instruktiven Einleitungen und Zwischenbemerkungen versehen, die ihre Einordnung ermöglichen. In die Texte sind immer wieder Hinweise auf Kommentare und weiterführende Literatur eingeschoben. Durch die Verschränkung von Quellentexten und Erläuterungen des Herausgebers ist ein lebendiges Bild von der Entwicklungsgeschichte des Dialoggedankens und der Dialogpraxis in der römisch-katholischen Kirche entstanden.

Die Sammlung gliedert sich in fünf Teile: Im ersten werden Dokumente des Zweiten Vatikanischen Konzils präsentiert. Über »Lumen gentium« und »Nostra aetate« hinaus stellt F. auch das Missionsdekret »Ad gentes«, die Pastoralkonstitution »Gaudium et spes« und die Erklärung über die Religionsfreiheit "Dignitatis humanae« vor. Dadurch wird die religionstheologische Thematik in den breiteren Kontext missionsund pastoraltheologischer Positionsbestimmungen eingeordnet und in Beziehung zur Neubestimmung des Verhältnisses von Religion(en), Gesellschaft, Staatsrecht und politischer Autorität gesetzt.

Die folgenden drei Teile sind den Stellungnahmen der drei Päpste Paul VI., Johannes Paul II. und Benedikt XVI. gewidmet. Wie zu erwarten nehmen die Aussagen Johannes Paul II. den weitaus größten Raum ein. Ihrer Fülle wegen sind sie nicht chronologisch, sondern thematisch geordnet - nach den Religionen, an deren Vertreter er sich in seinen Ansprachen wandte. In ihnen zeigt sich die Offenheit des Papstes für andere Religionen auf eindrucksvolle Weise. Ohne jegliche Relativierung der eigenen Wahrheitsgewissheit - im Gegenteil: als Frucht dieser Gewissheit - proklamiert er den Dialog als Beziehungsform. Die Notwendigkeit dazu ergibt sich für ihn nicht zuerst aus den Notwendigkeiten der friedlichen Koexistenz der Religionsgemeinschaften, sondern aus der Wesenbestimmung Gottes: »Er ist ein Gott des Dialogs, der seit den Uranfängen für ein Heilsgespräch mit der Menschheit, die er geschaffen hat, eintritt, ein Gespräch, das auch heute stattfindet und bis ans Ende aller Zeiten fortgeführt werden wird« (86). Daraus folgt für den Papst: »Durch Dialog lassen wir Gott in unse- 
rer Mitte gegenwärtig sein; denn wenn wir uns einander im Dialog öffnen, öffnen wir uns auch für Gott« (85).

Im letzten Teil hat F. Erklärungen der Dikasterien (Zentralbehörden, Ämter) der Römischen Kurie zusammengestellt, die unter Bezugnahme auf die Erklärungen des Zweiten Vatikanischen Konzils und die Äußerungen der Päpste Interpretationen, Präzisierungen, Klarstellungen zu religions- und missionstheologischen Themen bieten. Dort finden sich auch Texte, die vor den vermeintlichen Gefahren einer zu weit gehenden, das heißt die Einzigartigkeit des Christusgeschehens, die Universalität des darin vermittelten Heils und die Heilnotwendigkeit der Kirche nicht deutlich genug betonenden Religionstheologie warnen und Grenzen ziehen, wie etwa die umstrittene Erklärung »Dominus Iesus« oder auch die Maßregelung von Jacques Dupuis in Form einer Notifikation zu seinem Buch »Towards a Christian Theology of Religious Pluralism «. ${ }^{3}$ Der Band dokumentiert also nicht nur die Öffnung der katholischen Kirche für die Religionsbegegnung und ihre theologische Reflexion, sondern auch die Beschränkung der davon ausgelösten Dynamik und die Auseinandersetzung darum. Kirchenpersonalpolitisch schlägt sich diese nieder in Abmahnungen sowie Verweigerungen und Entzügen der Lehrerlaubnis (524). Der Geist des offenen Dialogs mit Angehörigen anderer Religionen steht zuweilen in eklatantem Gegensatz zu den Kommunikationsformen innerhalb der katholischen Kirche. Es sind keine herrschaftsfreien, sondern machtförmige Kommunikationen. Auf der Grundlage solcher ungleicher Machtverteilungen kann sich aber kein echter Dialog entfalten.

Wie Ulrich Dehn so hat auch F. bei der Auswahl der zu dokumentierenden Texte Grenzen gezogen. Stellungnahmen der katholischen Kirche zum Judentum und zu den jüdisch-christlichen Beziehungen sind in seinem ausgezeichneten Reader unterrepräsentiert, weil sie andernorts bereits editiert sind. ${ }^{4}$ Fast zeitgleich mit dem von ihm herausgegebenen Band erschien die von CIBEDO (»Christlich-islamische Begegnungs- und Dokumentationsstelle« der Deutschen Bischofskonferenz) besorgte Zusammenstellung der offiziellen Dokumente der katholischen Kirche zum Dialog mit dem Islam, die ebenfalls von Kardinal Lehmann angeregt worden ist. Als Vorlage diente die französische Fassung der o.g. Publikation des Päpstlichen Rates für den Interreligiösen Dialog »Le dialogue interreligieux dans l'enseignement officiel de l'Église catholique. Du Concile Vatican II à Jean-Paul II (1963-2005)«. Unter Mitwirkung von Peter Hünseler und Christian W. Troll, der die Texte zum Islam im zweiten Teil ausgewählt und die Einleitung verfasst hat, wurde der Band von Timo Güzelmansur editiert.

${ }^{3}$ Dt.: Unterwegs zu einer christlichen Theologie des religiösen Pluralismus, hg. von Ulrich Winkler, übersetzt von Sigrid Rettenbacher (Salzburger Theologische Studien interkulturell 38), Innsbruck 2010.

4 Die Kirchen und das Judentum. Bd. 1: Dokumente von 1945-1985, hg. von Rolf Rendtorff, Paderborn 1988; Bd. 2: Dokumente von 1986-2000, hg. von Hans Hermann Henrix, Paderborn 2001. 
Der Band besteht aus zwei Teilen. Im ersten Teil, der weit mehr als die Hälfte des Volumens einnimmt, werden - wie bei Fürlinger - Grundsatzerklärungen zum interreligiösen Dialog im Allgemeinen vorgestellt: die Erklärungen des Zweiten Vatikanischen Konzils, wobei hier auch kurze Passagen aus »Dei Verbum« und »Apostolicam Actuositatem« Berücksichtung finden, päpstliche Lehrschreiben, nachsynodale apostolische Schreiben, päpstliche Ansprachen und Botschaften (von Paul VI. bis Benedikt XVI.), Dokumente der Dikasterien der Römischen Kurie, kirchenrechtliche Bestimmungen und das wichtige Papier der Internationalen Theologenkommission »Das Christentum und die Religionen« aus dem Jahre 1996.

Im zweiten Teil sind dann Texte versammelt, die sich unmittelbar auf den Dialog mit dem Islam beziehen. Durch die kritische Aufmerksamkeit, die dem Islam in jüngerer Vergangenheit in der öffentlichen Wahrnehmung zuteil geworden ist, ist gerade dieser Dialog von besonderer Bedeutung. Zunächst wird der Blick wiederum zurück zu den epochemachenden Aussagen des Zweiten Vaticanums gelenkt. Dann werden analog zum ersten Teil - die nachsynodalen apostolischen Schreiben Johannes Pauls II. und schließlich die Ansprachen und Botschaften der Päpste wiedergegeben. Im letzten Abschnitt des zweiten Teils sind die aktuellen Texte der Deutschen Bischofskonferenz (2003-2008) abgedruckt. Der letzte dieser Texte ist dem Bau von Moscheen in Deutschland gewidmet. Vor dem Hintergrund der Schweizer Entscheidung, ein Bauverbot für Minarette verfassungsrechtlich zu verankern, hat er besondere Bedeutung. Darin unterstreicht die Bischofskonferenz das Recht der Muslime auf den Bau würdiger Moscheen und verurteilt alle Versuche, „Sorgen und Ängste der Bevölkerung politisch zu instrumentalisieren. Kritik ist erlaubt, Hetze nicht« (Abs. 2602).

Hinsichtlich der Textauswahl überschneidet sich die Quellensammlung weitgehend - besonders im ersten Teil - mit dem von Fürlinger herausgegebenen Band. Während dort aber durch die intensive Kommentierung eine spannend zu lesende Mischung aus Quellenedition, Einführungswerk und Übersichtsdarstellung entstanden ist, hat CIBEDO eine reine Materialsammlung vorgelegt. Bis auf die knappe Einleitung von Chr.W. Troll (»Katholisches Lehramt und Islam seit dem Konzil«) gibt es keine Informationen zur Kontextualisierung und keine Anleitung zur Interpretation der Texte. Gerade aber die zahlreichen Ansprachen und Botschaften der Päpste, die der Band enthält, lassen sich in Inhalt und Sprachgestalt nur im Bezug auf die jeweiligen Kontexte verstehen, in denen sie vorgetragen wurden. Die Orte, Adressaten und zum Teil auch die Anlässe der Reden sind aus den Titeln zu ersehen. Die Situationen, in die hinein sie sprechen, bleiben jedoch im Dunkeln.

Als Materialsammlung hat der Band dennoch einen hohen Wert. Durch die Absatznummerierungen und die Register im Anhang eignet er sich gut als Nachschlagewerk für die Suche nach Quellen, deren Auswertung und Zitation. Ein programmatischer Impuls wie bei Fürlinger mit seinem Plädoyer für die Fortsetzung der Dialogbemühungen ist hier nicht zum Ausdruck gebracht. Er ist aber in der Intention für die Edition des Bandes enthalten und 
klingt in der bedenkenswerten Charakterisierung des interreligiösen Dialogs an, die Kardinal Lehmann in seinem Vorwort zu diesem Band gibt: »Im interreligiösen Dialog geht es insgesamt nicht in erster Linie um den Gewinn einer oft abstrakt bleibenden Gemeinsamkeit, sondern um die Einübung in ein Leben in gegenseitiger Achtung und um das Erlernen der Identität des Anderen« (18). Dass er sich ein beherzteres Voranschreiten auf diesem Weg wünscht, hatte er in seinem Vorwort zu Fürlingers Band zu erkennen gegeben: »Wir sind zumal im deutschen Sprachgebiet der damit vielfach beschriebenen Aufgabe noch längst nicht gerecht geworden« (10).

Die beiden - sich in ihrer konzeptionellen Verschiedenheit gegenseitig gut ergänzenden - Bände von Fürlinger und CIBEDO beziehen sich auf die offiziellen Texte des römischen Offiziums. Ebenso dokumentierenswert wären daneben die Stellungnahmen von Ortskirchen besonders in Ländern, in denen Christen mit Angehörigen anderer Religionen zusammenleben, etwa in Indien, Indonesien oder in weiten Teilen Afrikas. Dies würde noch einmal ein anderes Bild ergeben, vor allem in religionstheologischer Hinsicht. Theologen, die aus Indien stammen oder lange dort gelebt und gelehrt haben - wie Jacques Dupuis - betonen vielfach, dass die in Christus manifestierte Gegenwart Gottes in seinem Wort und Geist in den Manifestationen des kirchlichen Christentums nicht aufgeht, sondern sich auch in anderen Ausdrucksgestalten symbolisieren kann. Dagegen hatte sich »Dominus Iesus« gewendet. Im Sinne eines intrachristlichen religionstheologischen Dialogs wäre ein Reader nützlich, der alternative Positionen zu denen des Lehramts sammelt und zur Diskussion stellt.

Eine andere Gruppe von Dokumentationen bezieht sich nicht auf Texte, sondern auf Projekte und die darin gesammelten Erfahrungen. Als ersten Band der neuen Reihe »Religionen im Dialog« hat Wolfram Weiße eine Textsammlung mit dem Titel »Theologie im Plural« herausgegeben. Darin sind Initiativen und Institutionen vorgestellt, die außerchristliche Theologien im universitären Kontext etabliert und auf diese Weise »wissenschaftliche Ressourcen in Forschung und Lehre für den Bereich einer Theologie der Weltreligionen« (7) geschaffen haben. Wenn von einer unaufhebbaren, weil nicht auf eine letzte Einheit rückführbaren Vielfalt der Religionen auszugehen sei, müsse auch den theologischen Selbstreflexionen dieser Traditionen Raum gegeben werden. Die kulturwissenschaftlichen außenperspektivischen Zugänge der Religionswissenschaft, der Islamwissenschaft, der Indologie usw. sollten vermehrt durch innenperspektivische Artikulationen der Religionstraditionen in Forschung und Lehre ergänzt werden. Diese Forderung wird untermauert durch den Bedarf an Einrichtungen zur Ausbildung von Lehrkräften für den schulischen Religionsunterricht in außerchristlichen Tra- 
ditionen und zur Qualifikation von intellektuellen, administrativen und spirituellen Führungspersönlichkeiten für die Leitung ihrer Religionsgemeinschaften. Diese Notwendigkeit hat sich in den letzten Jahren in Westeuropa vor allem in der Installation von Lehrstühlen für islamische Theologie an den Universitäten niedergeschlagen. Ein großer Teil des Bandes ist der Vorstellung dieser Einrichtungen gewidmet.

Der erste Teil (»Internationale Perspektiven«) enthält drei Beiträge, die weniger dokumentarischer und mehr reflexiver Art sind. Peter L. Berger schlägt vor, die Säkularisierungstheorie durch eine Pluralisierungstheorie zu ersetzen. Der religiöse Pluralismus sei eine unausweichliche Folgeerscheinung des Modernisierungsprozesses und der Migrationsbewegungen, die eine Verdichtung der Kommunikationsnetze und die Anhebung der Bildungsstandards mit sich bringe. Dadurch werde die selbstverständliche Akzeptanz der Religionen untergraben. Religion sei immer weniger Schicksal und immer mehr Wahl. Darin klingt Bergers bekannter Gedanke vom »Zwang zur Häresie« an. ${ }^{5}$ Doch dürfe die Individualisierung der Religion nicht zur Vergleichgültigung der gewählten Optionen führen; schon deshalb nicht, weil jede Gesellschaft auf einen Minimalkonsens bezüglich grundlegender Werte und Prinzipien angewiesen sei. »Ich glaube, wir brauchen ein gutes Stück Selbstsicherheit in Religion, Moral und Politik, ohne dabei zu Fundamentalisten, Fanatikern und Fremdenhassern zu werden« (19).

Der Religionswissenschaftler Zhuo Xinping gibt einen instruktiven Überblick über die Religionsgeschichte Chinas, die gegenwärtigen Entwicklungen der chinesischen Religionslandschaft und Ansätze zum interreligiösen Dialog. Dabei lässt sich erkennen, wie sehr das Verständnis des interreligiösen Dialogs mit dem jeweiligen geisteskulturellen Kontext verschränkt ist. Der für die (vom Konfuzianismus geprägte) Kultur Chinas zentrale Gedanke der Harmonie bestimmt auch die Leitvorstellungen zur Gestaltung der interreligiösen Beziehungen. Harmonie bedeute im Konfuzianismus eine »anpassende Gemeinsamkeit, nicht aber Gleichheit« (31). Diese Vorstellung ließe sich Xinping zufolge mit einer »Theologie des Regenbogens « in Verbindung bringen, die auf jüdischer und christlicher Seite im Anschluss an Gen 9,13-17 entwickelt werden könnte.

In interessantem Kontrast dazu steht der Vorschlag des jüdischen Theologen Ephraim Meir, die Beziehungen zwischen den Religionen - wobei er allerdings nur die rabrahamischen Religionen im Blick hat - durch den Gedanken und die Praxis der gegenseitigen Gastfreundschaft - im Bewusstsein der gemeinsamen Abstammung - zu bestimmen. Während die chinesische Religionskultur auf die individuelle Spiritualität fokussiert ist und die Heiligung und Vervollkommnung der Seele betont (»Religion des Herzens« statt »Religion der Form«, 29), spielt für die Sprösslinge des semitischen Religionsstammbaums die Gesellschaftsgestaltung eine wichtige Rolle. Die in den Wurzeln der drei Religionen liegenden zivilisatorischen Potenziale sollten - so Meir - für die Schaffung und Erhaltung der friedlichen Koexistenz auf allen Ebenen des interkulturellen und interreligiösen Zusammenlebens in der Gesellschaft und zwischen den Gesellschaften fruchtbar gemacht werden. Dazu sei die Ausbildung eines »exilischen

${ }^{5}$ P.L. Berger, Der Zwang zur Häresie. Religion in der pluralistischen Gesellschaft, Frankfurt a.M. 1980, engl. The Heretical Imperative. Contemporary Possibilities of Religious Affirmation, New York 1980. 
Denkens « erforderlich, das nicht auf sich selbst fixiert ist und auf den Wahrheits- und Heilsbesitz der eigenen religiösen Traditionen pocht, sondern das »Zentrum außerhalb des Selbst lokalisiert« (39) - in der Bindung an die transzendente Realität Gottes, die religiöse Grenzen überschreitet.

Im zweiten Teil des Bandes werden Initiativen zur Etablierung außerchristlicher schwerpunktmäßig islamischer - Theologie auf Hochschulebene in Europa vorgestellt: die islamisch-theologische Fakultät in Sarajevo, das Zentrum für islamische Theologie an der Freien Universität Amsterdam, die islamische Universität Rotterdam, der »Private Studiengang für das Lehramt für Islamische Religion an Pflichtschulen« in Wien. Hinzu kommen zwei Artikel, in denen allgemeinere Betrachtungen zu islamischer Bildung im Kontext Italiens und zum Verhältnis von Religionswissenschaften und Theologien an den Universitäten aus französischer Perspektive angestellt werden. Dabei lassen die Autoren auch wissenschaftstheoretische Überlegungen anklingen, die für die kritische Selbstreflexion islamischer Forschung und Lehre und ihre Positionierung an der Universität von Bedeutung sind. So forderte etwa Jean-Paul Willaime in seinem Beitrag, dass die islamische wie jede Theologie folgende fünf Bedingungen erfüllen müsse: Offenheit für religiöse Pluralität, Ausbildung eines kritisch-hermeneutischen Ansatzes, Zugänglichkeit für alle Studierenden, Unabhängigkeit von religiösen Organisationen, die Besetzung der Stellen mit Vertretern der jeweiligen Religion, die eine authentische Innenperspektive vertreten können.

Im dritten Teil des Bandes sind Initiativen aus Deutschland vorgestellt: das Zentrum für Interreligiöse Studien an der Universität Bamberg, die Stiftungsprofessur für Islamische Religion an der Universität Frankfurt, das Centrum für Religiöse Studien an der Universität Münster, die islamische Religionspädagogik an der Universität Osnabrück, die Ausbildung in islamischer Religionslehre an der Universität Erlangen-Nürnberg und das Interdisziplinäre Zentrum Weltreligionen im Dialog an der Universität Hamburg. In einem weiteren Beitrag wird die Islamkunde in Nordrhein-Westfalen als religionspädagogischer Modellversuch präsentiert. Alle diese Projekte sind mit spannenden Prozessen der Konzeptentwicklung verbunden. In ihnen wird um die Frage gerungen, wie eine Religionspädagogik - sowohl für die Ausbildung der zukünftigen Lehrerinnen und Lehrer als auch für den schulischen Islamunterricht - beschaffen sein sollte, die sowohl dem Selbstverständnis der islamischen Traditionen als auch den Standards der deutschen Bildungslandschaft angemessen ist. Was es für die Entfaltung der islamischen Theologie an deutschen und europäischen Universitäten bedeutet, dass sie sich von vorneherein in eine Situation gestellt sieht, die durch den Pluralismus christlicher und zum Teil auch jüdischer Theologien sowie durch die Nachbarschaft zu religions- und islamwissenschaftlichen Disziplinen geprägt ist, wird die Zukunft zeigen.

In einem Anhang sind Dokumente zu einigen der vorgestellten Projekte enthalten. Diese Strukturberichte und Studienpläne vermitteln einen Einblick in die Curricula und ihre didaktischen Leitvorstellungen.

Insgesamt gibt der Band einen interessanten Einblick in die Bewegung, die nicht nur in die akademische und schulische Religionspädagogik, sondern auch in die bisher übliche Disziplinenaufteilung von (christlicher) Theologie und Religionswissenschaft an einigen Universitäten gekommen ist. Die Etablierung islamischer Theologie im europäischen Wissenschaftsbetrieb wird 
sich auf das Selbstverständnis und die internen Diskurse in den muslimischen Gemeinschaften auswirken, und sie wird die christliche Theologie vor die Aufgabe stellen, ihre Rolle als Theologie unter Theologien neu zu bestimmen. Wenn sie diese Chance konstruktiv ergreift, kann es in Zukunft vermehrt zu akademischen Religionsdialogen kommen, von denen alle Beteiligten profitieren.

Neben dem interreligiösen Dialog, der schon seit den 70er Jahren des 20. Jh.s auf vielen Ebenen - auf der Ebene der Kirchen bzw. Religionsgemeinschaften, der Akademien und wissenschaftlicher Institutionen zum Zweck der Beziehungspflege, der praktischen Kooperation und des intellektuellen Austauschs - stattfindet, hat in den letzten Jahren der Dialog zwischen Politik und Religion an Bedeutung gewonnen. Vor dem Hintergrund der stärkeren öffentlichen Wahrnehmung der Religion im Allgemeinen und des Islam im Besonderen, hatte die neu gewählte Deutsche Bundesregierung im Jahre 2005 in ihrem Koalitionsvertrag einen intensiven Dialog mit den großen christlichen Kirchen, mit Juden und Muslimen vereinbart. Dem Dialog mit dem Islam wurde dabei besondere Relevanz beigemessen. Er sollte Teil der Integrationspolitik sein und der Ausbreitung von Rassismus, Extremismus und Antisemitismus entgegen wirken. Um diese Vereinbarung umzusetzen, rief Innenminister Wolfgang Schäuble am 27. September 2006 die »Deutsche Islam Konferenz« (DIK) ins Leben.

Ihre Aufgabenstellung, ihre Struktur und Arbeitsformen und vor allem die Resultate, die sie bis zum Jahr 2009 hervorgebracht hat, sind in einem Band dokumentiert, der - gefördert vom Bundesministerium des Innern - von der DIK herausgegeben worden ist. Als Untertitel trägt er das programmatische Leitwort der Konferenz: »Muslime in Deutschland - deutsche Muslime«. Die Dokumentation enthält nicht nur Grundsatzreden, Informationen zur Zusammensetzung und zur Arbeit der Konferenz sowie zu Projekten des interreligiösen Dialogs und der Integrationsförderung, sondern auch ausgewählte Vorträge von Mitgliedern der DIK, die aktuelle und zumeist auch wissenschaftlich fundierte Einblicke in den sgelebten Islamı in Deutschland geben. Damit stellt sie wichtiges Material auch für die akademische Reflexion der christlich-islamischen Beziehungen zur Verfügung.

Die erste Arbeitsgruppe befasste sich mit grundsätzlichen Fragen der Gesellschaftsordnung, der Grundrechte und der sie tragenden Werte. Dabei ging es zentral um die politische Partizipation der Muslime an einer demokratischen Gesellschaft, die auf dem Prinzip der Säkularität und der relativen Trennung von Religion und Politik gründet, aber auch um Wertevermittlung in der Familie und um die Gleichberechtigung der Geschlechter. Im Rahmen dieser Arbeitsgruppe wurde ein Forschungsprojekt lanciert, das aussagekräftige Daten zum Islam in Deutschland erheben sollte (s.u.). 
Doch beschränkte sich diese Arbeitsgruppe keineswegs auf die Beschreibung und Analyse der gegenwärtigen Situation. Es wurden - wie es der Gründungsauftrag der DIK vorsieht - auch kritische Fragen von grundsätzlicher Bedeutung aufgeworfen. Tilman Nagel etwa konfrontiert die muslimischen Teilnehmer der Konferenz mit kritischen Überlegungen zur Kompatibilität des Islam mit der geisteskulturellen Verfasstheit der Neuzeit in Europa. Dazu gehört die Selbstbehauptung des Menschen gegen religiöse Autorität. Säkularität verlange den Verzicht auf die Durchsetzung absoluter Wahrheitsansprüche. Leider dokumentiert der Band nicht die Diskussion, die sich an die Vorträge anschloss.

Die zweite Arbeitsgruppe wandte sich den religionspraktischen Angelegenheiten zu: Islamischer Religionsunterricht an den Schulen, Teilnahme muslimischer Kinder an schulischen Veranstaltungen (koedukativer Schwimmunterricht, Sexualkundeunterricht, Klassenfahrten), Moscheebau, Bestattungspraxis. Wie können für die damit angesprochenen Schwierigkeiten Lösungen im Rahmen der deutschen Rechtsordnung gefunden werden? Die Arbeitsgruppe hat bereits ein Grundlagenpapier für die Einführung islamischen Religionsunterrichts in deutscher Sprache vorgelegt.

Die dritte Arbeitsgruppe befasste sich mit dem Beitrag, den Wirtschaft und Medien zur Integrationsförderung der Muslime leisten können. Sie arbeitete das in den Medien vermittelte Islambild auf, erörterte Fragen der Berufsausbildung und des Arbeitsmarktes und initiierte Projekte, die eine bessere Eingliederung muslimischer Bürger in die ökonomischen Systeme der Gesellschaft zum Ziel haben.

In den Plenarsitzungen wurden die Ergebnisse und Empfehlungen der Arbeitsgruppen beraten und die Schritte auf dem weiteren Weg festgelegt. Auf der dritten Plenarsitzung wurden u.a. Empfehlungen zu folgenden Themenbereichen verabschiedet: »Integration von Muslimen in die deutsche Gesellschaftsordnung und die Werteordnung des Grundgesetzes«, »Einführung eines konfessionellen islamischen Religionsunterrichts in deutscher Sprache an öffentlichen Schulen«, »Bau und Betrieb von Moscheen", »Berichterstattung in Medien" und »Gemeinsames Vorgehen gegen gewalttätige und extremistische Bestrebungen islamistischer Vereinigungen«. Der letztgenannte Themenkreis wurde in einem eigens dafür eingerichteten »Gesprächskreis Sicherheit und Islamismus« erörtert.

Der Band stellt einen wichtigen und wegweisenden Beitrag zum Dialog zwischen den politisch Verantwortlichen des deutschen Staates und führenden Vertretern der Muslime in Deutschland dar, der auch für den christlichislamischen Dialog von Bedeutung ist. Denn die Fragen, um die es in diesem Dialog geht, betreffen zum großen Teil die Präsenz des Islam in der Öffentlichkeit. Viele der dabei auftretenden Integrationsprobleme sind allerdings weniger religiös als kulturell bedingt.

Die im Auftrag der DIK und mit Unterstützung des »Bundesamtes für Migration und Flüchtlinge" erarbeitete Studie »Muslimisches Leben in Deutschland«, herausgegeben von Sonja Haug, Stephanie Müssig und Anja STichs, bietet eine wissenschaftliche Informationsquelle zum Islam in Deutschland. Sie dokumentiert die Resultate eines Forschungsprojekts, bei dem Erkenntnisse über das religiöse Leben, und die soziale und strukturelle 
Integration muslimischer Migranten gewonnen werden sollten. Dazu wurde eine Fülle quantitativer und (durch telefonische Befragung von 6000 Personen) auch qualitativer Daten gesammelt und ausgewertet. Die statistischen Daten und die Untersuchungen zur religiösen Praxis der Muslime in Deutschland lassen erkennen, wie sich die Religionszughörigkeit und auch die regionale Herkunft der aus 49 Herkunftsländern stammenden Muslime auf die Prozesse ihrer Integration auswirken. Aus Vergleichen mit Migrantengruppen anderer Religionszugehörigkeit geht hervor, dass »Muslime bei verschiedenen Indikatoren niedrigere Integrationswerte auf(weisen) als Angehörige anderer Religionen aus den selben Herkunftsländern« (15). Andererseits konnte eine explizite Abgrenzungstendenz nicht festgestellt werden. Der Anteil derer, die keine Kontakte zu Deutschen haben und auch nicht haben wollen, beträgt lediglich 1\% (17).

Nach der Einleitung, in der die Projektleiter den Forschungsstand referieren und Rechenschaft über die Methode ihrer Untersuchung geben, wird im zweiten Kapitel die Zahl der in Deutschland lebenden Muslime ermittelt. Sie liegt mit 3,8 bis 4,3 Mio höher als nach bisherigen Schätzungen angenommen wurde. Im zweiten Teil dieses Kapitels gibt die Studie Aufschluss über die Zusammensetzung dieser Bevölkerungsgruppe im Hinblick auf Glaubensgemeinschaften, Alter, Geschlecht und Verteilung auf die Bundesländer. Das dritte Kapitel präsentiert soziodemographische und migrationsbiographische Untersuchungsergebnisse, bei denen die Herkunftsländer, Alter, Geschlecht und Religion (3.1.) sowie Generationenzugehörigkeit, Aufenthaltsdauer und Einwanderungsalter (3.2.) in Korrelation zueinander gesetzt werden. Aufschlussreich ist auch die Erhebung der Einwanderungsgründe (3.3.), der Staatsangehörigkeiten und Einbürgerungswege (3.4.), der Haushaltsgröße und Kinderzahl (3.5.).

Besondere Aufmerksamkeit verdient das vierte Kapitel, in dem die Religiosität und die religiöse Praxis der Muslime behandelt werden. Nicht nur die quantitative Verteilung der unterschiedlichen Glaubensrichtungen wird dargestellt, sondern auch die subjektive Einschätzung der eigenen Religiosität: 36\% bezeichnen sich als »sehr stark gläubig«, $50 \%$ als »eher gläubig«, $10 \%$ als »eher nicht gläubig« und $4 \%$ als "gar nicht gläubig«. Die Angaben schwanken allerdings nach Herkunftsländern. Der Anteil der stark Gläubigen liegt bei den aus Südosteuropa zugewanderten Muslimen signifikant unter $36 \%$, bei türkischstämmigen dagegen bei $40 \%$. Bei Muslimen aus dem Iran liegt die Selbsteinschätzung, gar nicht oder eher nicht gläubig zu sein, deutlich über dem statischen Durchschnitt aller Muslime. Im Blick auf die religiöse Praxis wurden die Gebetshäufigkeit, das Begehen von Festen, die Speise- und Festgewohnheiten, die Teilnahme an religiösen Veranstaltungen erfragt. Daraus - wie auch aus dem Engagement in Kulturvereinen - ergeben sich Rückschlüsse auf das »soziale Kapital« (die Entwicklung von Organisations- und Kommunikationsfähigkeiten). Im Abschnitt 4.6. gibt die Studie Auskunft über die Bekanntheit islamischer Verbände unter den Muslimen und die Identifikation mit ihnen. Dieser Teil der Erhebung ist nicht zuletzt deshalb wichtig, weil sich daraus erkennen lässt, wie gut sich die Muslime in der DIK repräsentiert sehen. Detaillierte Auskunft über die Teilnahmen muslimischer Kinder an schulischen Angeboten gibt Abschnitt 4.7. 
Im fünften Kapitel sind Daten zusammengestellt, die Fragen der Integration betreffen - in 5.1. hinsichtlich der strukturellen und kognitiven Integration (Bildung, Erwerbstätigkeit, Stellung im Beruf, Einkommen, Sprachkompetenzen, Teilnahme an Integrationskursen) und in 5.2. hinsichtlich der sozialen Integration (Mitgliedschaft in Organisationen und Vereinen, interethnische Kontakte, interreligiöse Offenheit, Wohnungsumgebung, Verbundenheit mit Deutschland und dem Herkunftsland). Profile von muslimischen Migrantengruppen, spezifiziert nach Herkunftsregionen, sind im sechsten Kapitel präsentiert, wobei hier - wie auch sonst in der Studie - die Aleviten gesondert ausgewiesen sind. Am Schluss des Bandes steht eine Zusammenfassung der Ergebnisse, ein Literaturverzeichnis und eine Sammlung der verwendeten Fragebögen.

Der Band gibt einen detaillierten Einblick in die facettenreiche Lebenswelt, das Selbstverständnis und die religiöse Praxis der Muslime in Deutschland. Mit dem differenzierten Bild, das sich dabei abzeichnet, trägt er wesentlich zur Versachlichung der Debatten um die gesellschaftliche Integration dieser Migrantengruppen bei. Es zeigt sich, dass die Religiosität zwar ein wichtiger Faktor bei der Ausgestaltung des Lebens muslimischer Bürger darstellt, dass sie aber nicht überbewertet werden darf. Andere Faktoren spielen für das Selbstbild der Muslime und damit für ihre Teilhabe am gesellschaftlichen Leben in Deutschland eine ebenso wichtige Rolle. Daraus ziehen die Verfasser der Studie den Schluss, dass Integrationsangebote, die sich speziell an religiöse Muslime richten, nicht für die Zielgruppe als ganze geeignet sind. Integration durch Sprache und Bildung sollte besonders gefördert werden. Die soziale Integration der Muslime ist nach Auskunft der Studie aber besser, als es die öffentliche Diskussion zu diesem Thema vermuten lässt. Für die Gruppe der türkischen Muslime wurden im Vergleich zu anderen Einwanderergruppen jedoch Defizite im Bezug auf die strukturelle Integration festgestellt. Gründe dafür sind im durchschnittlich niedrigen Bildungsniveau, im Wohnumfeld (Gegenden mit hohem Ausländeranteil) und in der geringer ausgeprägten sozialen Vernetzung mit Deutschen zu suchen.

Bemerkenswert ist die interreligiöse Offenheit der Muslime, die anhand der Bereitschaft ermittelt wurde, religionsverbindende Ehen einzugehen. 65\% der befragten Alleinstehenden und 58\% der Verheirateten können sich eine Partnerschaft mit einer Person anderen Glaubens vorstellen. Der Eheschließung des Sohnes mir einer Nicht-Muslima würden $80 \%$ zustimmen, für die Töchter liegt ein solches Einverständnis allerdings bei nur $63 \%$.

\section{Religionstheologische Studien}

Edmund Arens, Gottesverständigung. Eine kommunikative Religionstheologie. Herder, Freiburg i.Br. 2007, 272 S. - Reinhold Bernhardt / Perry Schmidt- 
Leukel (Hg.), Multiple religiöse Identität. Aus verschiedenen religiösen Traditionen schöpfen (Beiträge zu einer Theologie der Religionen 5). Theologischer Verlag, Zürich 2008, 340 S. - Reinhold Bernhardt / Klaus von Stosch (Hg.), Komparative Theologie Interreligiöse Vergleiche als Weg der Religionstheologie (Beiträge zu einer Theologie der Religionen 7). Theologischer Verlag, Zürich 2009, 349 S. - Uwe Gerber, Wie überlebt das Christentum? Religiöse Erfahrungen und Deutungen im 21. Jahrhundert. Erlösung - Versöhnung - Erleichterung - Vereindeutigung - Alterität (Beiträge zu einer Theologie der Religionen 4). Theologischer Verlag, Zürich 2008, 298 S. - Eiler t Herms, Zusammenleben im Widerstreit der Weltanschauungen. Beiträge zur Sozialethik. Mohr Siebeck, Tübingen 2007, XX + 457 S.

Wie schon in seinen früheren Publikationen geht Edmund Arens in seinem Buch »Gottesverständigung" davon aus, dass Religion zuerst und vor allem kommunikatives Handeln ist - ein Praxisvollzug, der erst sekundär reflektiert und in bekenntnis- und lehrhafte Formen gegossen wird. Eine »kommunikative Religionstheologie« hat demgemäß die Grundorientierungen und -formen dieses Handelns zu bestimmen sowie die mit ihnen verbundenen Geltungsansprüche herauszuarbeiten. Sie vollzieht sich aber auch in praktischer Absicht, denn es geht ihr nicht nur um Diagnose und Reflexion, sondern auch um eine Zurückdrängung der aggressiven und destruktiven Potentiale innerhalb und zwischen den Religionen. Sie will die Möglichkeiten der intra- und interreligiösen Verständigung ausloten und damit einen Beitrag zur friedlichen Koexistenz der Religionen und Kulturen leisten.

Interessant an diesem Ansatz ist die Mittelstellung zwischen Theologie und Religionswissenschaft, in der er sich lokalisiert. Wie die Religionswissenschaft nimmt er eine Außenperspektive auf Religion ein, anders als diese will er sich jedoch nicht auf Distanz zu den inneren Gestaltungsprozessen der Religionspraxis und zum Begegnungsgeschehen zwischen den Religionen halten, sondern Einfluss darauf nehmen. Die religionstheologischen Überlegungen sind gestützt auf einen breiten religionstheoretischen Unterbau, der unmittelbar an religionsphilosophische, religionssoziologische und religionspsychologische Diskurse anknüpft und das Gespräch mit ihnen sucht.

Im ersten Teil stellt A. religionsphilosophische und sozialwissenschaftliche Ansätze dar, die Religion als kommunikative Praxis thematisieren: die funktionalen Religionstheorien Lübbes und Luhmanns, die sozialanthropologisch ansetzenden ritualtheoretischen Zugänge von Arnold von Gennep, Victor Turner, Caterine Bell und Roy Rappaport. Schließlich zeichnet er die Entwicklung der Religionsdeutung bei Jürgen Habermas nach. Dessen Ansatz einer kommunikativen Handlungstheorie, wie er ihn schon in seinem systematischen Hauptwerk »Theorie des kommunikativen Handelns« entfaltet hatte, ist für A. von besonderer Bedeutung. Dort hatte Habermas noch die Substitution der im Zuge von weltanschaulichen Rationalisierungsprozessen obsolet gewordenen Religion durch eine kommunikative Ethik in der Moderne beschrieben. Seit den 
achtziger Jahren begann er dann, die Religion als lebensweltliche Ressource zu entdecken. Religion und Vernunft stehen sich nun nicht mehr antithetisch gegenüber, so dass »dem einen Pol die Zukunft gehört, während der andere obsolet geworden ist und im Orkus der Geschichte verschwindet« (54), sondern sie sind auf wechselseitige - auch streitbare - Kommunikation über die elementaren Fragen des menschlichen Lebens angewiesen.

Der zweite Teil des Bandes nimmt Ansätze der Sprach-, Kommunikations- und Handlungstheorie auf und unterscheidet fünf Dimensionen von religiöser Kommunikation: die intersubjektive (bezogen auf die involvierten Subjekte - »wer?«), die inhaltliche (die zum Ausdruck kommenden Inhalte - »was?«), die kontextuelle (Ort, Zeit, Rahmenbedingungen - "wo? «), die mediale (Ausdrucksformen und Medien der Kommunikation, wie etwa Texte - »wie?«) sowie die intentionale (die damit verbundenen Absichten - »wozu?«). Dieses formale Analyseraster erlaubt es, die Vielfalt der religiösen Phänomene zu systematisieren und die spezifischen Eigenschaften der religiösen Kommunikation herauszuarbeiten.

Ausgestattet mit diesem Kategoriensystem unterscheidet A. im dritten Teil vier Formen des Religiösen in der Gegenwart, die man allerdings auch als vier Aspekte bzw. Beschreibungsformen des Religiösen, das heißt: als vier Diskurse über Religion auffassen kann. Ausgehend von der Feststellung, dass Religion in der Moderne - entgegen der Säkularisierungsthese - nicht zum Verschwinden gekommen ist, sondern vielfältiger und damit unübersichtlicher wurde, beleuchtet er zunächst die institutionelle bzw. organisierte Religion als Produkt gesellschaftlicher Differenzierungsprozesse, zweitens die Erscheinungsformen des postmodernen religiösen Individualismus, drittens den religiösen Fundamentalismus als deren Gegenpol und schließlich die öffentliche Religion im Rahmen der Zivilgesellschaft.

Im vierten Teil stehen die religionstheoretischen Reflexionen und Deutungen zur Debatte, wie sie in der Religionswissenschaft entwickelt worden sind. A. zeichnet zunächst die religionsphänomenologischen Ansätze nach und stellt dann das sich davon kritische abwendende kulturwissenschaftliche Paradigma dar, wie es die gegenwärtige Religionswissenschaft weitgehend bestimmt. Dabei unterscheidet er Religionstheorien, die Religion in einem weiteren sozialwissenschaftlichen und -philosophischen Rahmen thematisieren (Max Weber, Robert Bellah, Niklas Luhmann und Jürgen Habermas) und Religionstheologien, die sauf der Grundlage, im Rahmen und aus der Perspektive einer bestimmten Religion« (187) entfaltet werden. A. stellt drei Argumentationslinien dar, auf denen sich die religionstheologische Reflexion im Christentum im 20. Jh. entwickelt hat: die religionsgeschichtlichen Ansätze von Ernst Troeltsch, Paul Tillich und Wolfhart Pannenberg, die heilsgeschichtliche Theologie Karl Rahners sowie die Entwürfe der sog. »Pluralistischen Religionstheologie« von John Hick, Wilfred Cantwell Smith und Paul Knitter.

Im fünften Teil präsentiert A. seinen eigenen Entwurf einer kommunikativ konzipierten Religionstheologie, die im Kontext der christlichen Glaubensgemeinschaft situiert ist. Ausgehend von einer Skizze seines Verständnisses von Religion als kommunikativer Praxis beschreibt er die Formen der im Christentum vollzogenen Glaubenskommunikation: Erzählen und Erinnern, Feiern und Verkündigen, Bezeugen und Bekennen, sowie das Teilen. Dann wendet er sich den Fragen zu, die in den religionstheologischen Debatten der Gegenwart erörtert werden: (a) Zum differenzierten Verständnis religiöser Geltungsansprüche greift er auf die Habermas'sche Unterscheidung von Wahrheit, Richtigkeit und Wahrhaftigkeit zurück. (b) Als Maßstäbe für die Beurteilung religiöser Geltungsansprüche schlägt er ein theologisches Kriterium (Ent- 
sprechung zur Bezeugung Gottes als kreativer und kommunikativer Wirklichkeit), ein christologisches Kriterium (Entsprechung zur kommunikativen Praxis Jesu) und ein ethisches Kriterium (Entsprechung zur Ethik des Mitleidens, der Gerechtigkeit, Solidarität und grenzüberschreitender Liebe) vor. (c) Hinsichtlich der Frage nach dem Standort und der Perspektive seines religionstheologischen Ansatzes gibt er zu erkennen, dass es sich dabei um ein eindeutig christlich-theologisches Unterfangen handelt. Darin unterscheidet es sich von der phänomenologischen Religionsforschung. Andererseits wahrt dieser Ansatz eine analytische und reflexive Distanz zum unmittelbaren Vollzug des Glaubens. "Eine kommunikative Religionstheologie legt keine Bekenntnisse ab, sondern reflektiert deren Dimensionen und Elemente, analysiert die Subjekte, Inhalte, Orte, Ausdrucksformen und Intentionen religiöser Bekenntnisse und expliziert deren Bedeutung für die Identität und Integrität von Glaubensgemeinschaften《 (253). (d) A.s kommunikative Religionstheologie zielt auf die wechselseitige differenzsensible Verständigung zwischen den religiösen Subjekten und Gemeinschaften, ist sich der Grenzen des Verstehens bewusst und lotet aus, inwiefern ein Einverständnis über die jeweiligen Überzeugungen und Handlungsorientierungen erzielt werden kann. Auf diese Zielrichtung verweist der Titel des Buches - „Gottesverständigung«.

A. hat mit diesem Buch die Fenster der (Religions-)Theologie zu handlungs- und kommunikationstheoretisch informierten Ansätzen der Religionsdeutung geöffnet, ohne dabei die Gebundenheit an die christliche Überlieferungsgemeinschaft preiszugeben. Aus dem Verständnis der Religion als kommunikativer Praxis ergibt sich mit innerer Folgerichtigkeit die Ausrichtung auf die interreligiöse Kommunikation. Die Fokussierung auf die religiöse Praxis zielt dabei jedoch nicht nur auf den »Dialog des Handelns«, sondern auf den Vollzug aller Erscheinungsformen des Religiösen. Auch die Auseinandersetzung über Glaubensinhalte ist Teil dieses Vollzugs. Die ideellen Gehalte der religiösen Traditionen sind immer eingebunden in Kommunikationsprozesse und nur aus deren Kontext heraus zu verstehen. Das Moment des Dialogischen ist damit schon in den Ansatz der kommunikativen Religionstheologie mit aufgenommen.

Wie Arens so geht auch Uwe Gerber von der Bestandsaufnahme der Religionslandschaft am Beginn des 21. Jh.s aus. Die traditionellen christlichen Religionsangebote sind in Mitteleuropa einer tiefgreifenden Transformation unterworfen: »Der Kern christlicher Religion verschiebt sich zusehends und deutlich vom Inhaltlichen zum Performativen, vom Rituellen zum Eventhaften, vom Zelebrieren zum Pragmatischen, vom Verbindlichen zum nützlichen Accessoire, von der Zumutung der Nächstenliebe zum Mediengerechten« (10). Auch die Verbindung mit außerchristlichen Religionsformen gehört dazu. Mit scharfem Pinsel zeichnet G. ein Bild dieser Veränderungen, bettet dieses Bild - wie Arens - in die gegenwärtigen Religionsdiskurse ein, nimmt auch die religionspolitischen Hintergründe in den Blick und fordert dann eine radikale post-christliche Revision des Christentumsverständnisses. 
Während Arens das Blickfeld ähnlich weit fasst, aber gerade daraus Impulse für eine christliche Religionstheologie gewinnt, geht G. über die Theologie der Religionen hinaus und visiert das »Projekt einer profanen Religiosität der Alterität« an. Er versteht es als Versuch, das Bonhoeffer'sche Programm des religionslosen Christentums unter den religionskulturellen Bedingungen der Gegenwart zu konkretisieren. Wie bei Bonhoeffer speist sich seine Kritik an einem metaphyischen Bezugsrahmen der Rede von Gott und seine Betonung der Weltlichkeit des Gottesglaubens letztlich aus dem Inkarnationsgedanken (dessen Ausdeutung an die Ansätze der »Gott-ist-tot-Theologie«, an Dorothee Sölle und an Herbert Braun erinnert). Wo die Selbstvergegenwärtigung Gottes in der Welt ernst genommen wird, muss G. zufolge die vertikale (platonisierende) Anordnung von Jenseits und Diesseits zugunsten einer »Horizontale(n) des Begegnens« von Ich und Du (26) aufgegeben werden.

Das Religionsverständnis, das sich daraus ergibt, hebt $G$. von vier Typen gelebter Religion ab, die er unter den Leitbegriffen »Erlösung - Versöhnung _ Erleichterung - Vereindeutigung« rekonstruiert. Mit dieser Typologie sollen »sozial-theologische Schneisen« (34) durch das Gestrüpp der wuchernden Religionsfauna geschlagen werden. Die ersten beiden dieser Typen stehen für die traditionelle römisch-katholische und protestantische Christentumsauffassung, die beiden letzten stellen die sich abstoßenden Pole der gegenwärtigen Religionskulturen dar. Diese Gegenüberüberstellung findet sich in ähnlicher Weise auch bei Arens. G. verbindet diese vier Typen durch das von J. Baudrillard entwickelte Dreier-Schema von Imitation, Produktion und Simulation miteinander, wobei er »Imitation« im Typus der Erlösungsreligion repräsentiert sieht, »Produktion« im Typus der Versöhnungsreligion und »Simulation« in den beiden Typen der Erleichterung und Vereindeutigung. In dieser Kombination dient ihm die Typologie sowohl zur Deutung der abendländischen Christentumsgeschichte wie zur Vermessung der gegenwärtigen Religionstopographie.

Der Religionstypus der Erlösung zielt auf die Befreiung des Menschen aus seinem sündig-sterblichen Körper und aus der gefallenen Welt. Heil wird im Aufstieg in die jenseitige Welt erreicht. G. sieht diesen Typus vor allem im vorreformatorischen Katholizismus realisiert. Ihm entspricht die Grundhaltung der gehorsamen »Imitation" tradierter kirchlicher Glaubensinhalte, Moralvorstellungen und Lebensformen.

Mit Reformation, Renaissance und Humanismus bricht sich nach G. ein Anthropozentrismus mit der Grundhaltung der »Produktion«Bahn. Der Mensch entdeckt sich selbst einschließlich seiner forschenden und weltgestaltenden Potentiale. Heilwerden besteht nicht mehr in der Erlösung aus der Welt, sondern in der »Versöbnung des Schöpfergottes mit seinen irdischen Mitgeschöpfen, indem er diese als seine Geschöpfe rechtfertigt und gegen ihre Sünde(n) gerecht spricht« (37). Damit sind sie zu eigenverantwortlicher Lebens- und Weltgestaltung befreit. 
Die Religionstypen der Erleichterung und der Vereindeutigung sind die charakteristischen Antipoden der Spätmoderne. Die individualistisch-egozentrische Erleichterungsreligion ist weder an Erlösung noch an der Gerechtsprechung durch Gott orientiert, sondern am gelingenden Leben, an Entlastung und spiritueller Geborgenheit. Um die Funktion der Sinnstiftung und Lebensbewältigung erfüllen zu können, reichert sie sich selektiv mit dem Traditionsgut der christlichen Erlösungs- und Versöhnungsreligion an, integriert aber auch Elemente aus anderen religiösen Traditionen. Ihr gegenüber steht die Tendenz zur Vereindentigung, die sich nach G. nicht nur im religiösen Fundamentalismus, sondern auch in den Medien niederschlägt, sofern es dort zur Ausblendung von Alterität und von differenzierenden Positionsbestimmungen kommt. In beiden Religionstypen sieht er die Grundhaltung der "Simulation « gegeben, in der das religiöse Subjekt medial vermittelte Inszenierungen des rechten Lebens rezipiert und aktualisiert. Es lebt im Netzwerk virtueller Zweitwelten und bringt die dort vorgefundenen Formate zur Darstellung.

Diesen vier Religionsformen stellt G. mit dem Typus der Alterität ein Verständnis von Religion gegenüber, das sowohl die Egozentrik der Erleichterungsreligion als auch die Entfremdung der Vereindeutigungsreligion hinter sich lässt und in dem er die Überlebenschance für das Christentum sieht. Es ist dadurch gekennzeichnet, dass »der Andere in seiner Einzigartigkeit, der Fremde in seiner Differenz, der Nächste in seiner Würde Anerkennung erfahren und ich ebenfalls diese Anerkennung, solches Anerkanntwerden als einzigartiges Geschöpf erfahre« (33). Auch darin mag man einen Niederschlag der Bonhoeffer'schen Formel »Kirche für andere«sehen.

Gegenüber der vom Subjekt zu leistenden Perfektionierung der Lebensmitte in den beiden Religionstypen der Erleichterung und der Entfremdung geht es im Typus der Alterität gerade umgekehrt um deren Freihaltung. Die Unverfügbarkeit und Entzogenheit des eigenen Selbstseins, seine (Vor-)Gegebenheit, sein Ausgesetzt- und Getragensein durch ein Anderes seiner selbst bestimmt die Grundhaltung. Autonomie, Identität und der Anspruch auf absolute Wahrheit werden bei dieser radikalen Selbstrelativierung obsolet. Das Bewusstsein radikaler Abhängigkeit führt in eine ebenso radikale Befreiung zur eigenverantwortlichen Lebensgestaltung. Es führt auch zur Anerkennung Anderer und zum wertschätzenden Umgang mit den natürlichen und kulturellen Rahmenbedingungen des Lebens.

Typologische Rekonstruktionen sind angreifbar, weil sie die Vielgestaltigkeit der Wirklichkeit auf Grundmuster reduzieren, die sich in dieser Einfachheit immer nur approximativ auffinden lassen. Und doch - oder gerade deshalb - haben sie Erschließungskraft. Wie schon von Max Weber in seiner Kennzeichnung der »Idealtypen« zugestanden, nehmen sie Überzeichnungen vor, um damit markante Gesichtzüge eines komplexen Phänomens herauszupräparieren und unterscheidbar zu machen. Diese Methode wendet auch 
G. an. Sie ist nicht an der detailgetreuen Abbildung der Phänomene interessiert, sondern an ihrer Charakteristik.

Es geht G. um eine spätmoderne Interpretation des Protestantismus. In der Entfaltung des von ihm favorisierten Religionstyps unternimmt er den Versuch, die in der reformatorischen Theologie verwurzelten Dialektik von schlechthinniger Abhängigkeit (vom Ganz-Anderen) und darin konstituierter endlicher Freiheit in einem postmetaphysischen, anerkennungsethischen Bezugsrahmen auszulegen. Von Gott kann dabei nur noch als dem Fremden und gänzlich Entzogenen gesprochen werden. Die Modi seiner Selbstvergegenwärtigung in Wort und Geist bleiben demgegenüber jedoch unterbestimmt. Gott wird zur Chiffre für die Gegebenheit und Unverfügbarkeit des Lebens und für die Zusage einer damit verbundenen Anerkennung, in der die Bedingung der Möglichkeit der Anerkennung anderer gründet. Doch wie soll diese Zusage erfolgen? Wie wird sie in der kommunikativen Praxis des Glaubens - um mit Edmund Arens zu reden - konkret?

Mit der Kritik an einer Doppelstöckigkeit der Wirklichkeit wird bei G. auch die eschatologische Ausrichtung hinfällig. Die Rechtfertigungsbotschaft bezieht sich nicht mehr auf Gottes letztgültiges Urteil über das Leben, sondern auf die Anerkennung des Menschen in der jeweiligen Gegenwart seines Lebensvollzugs. Sie wird in diesem Sinne sprotologisch ausgelegt.

Hier wie auch an vielen anderen Stellen bietet G.s De- und Rekonstruktion der protestantischen Christentumsauffassung wertvolle Anregungen. Aber sie bleibt monothematisch, entsubstantialisiert die christliche Tradition in erheblichem Umfang und entfernt sich damit weit von den kirchlich-theologischen Selbstverständigungsdebatten. Die Frage, wie ein solches Christentum als nachchristlich-profane Religion der Alterität aussehen soll, welche Sozialgestalten es annehmen und in welchen Vollzügen es sich realisieren soll, bleibt offen. Ob die angebotene Rezeptur das Überleben des Christentums zu sichern imstande ist, wie es der Titel des Buches insinuiert, bleibt daher fraglich.

Für den Religionsdialog ist dieser Ansatz insofern bedeutsam, als auch der religiös Andere unbedingte Anerkennung verdient. An seiner Religion aber besteht für G. wenig Interesse. Der Islam kommt im Kontext religionspolitischer Überlegungen und dann im Typus der Vereindeutigungsreligion zur Sprache. Dabei hätte ein theologischer Bezug zur Gott-Mensch-Beziehung in der islamischen Tradition im Lichte des Alteritätsgedankens durchaus reizvoll sein können. An anderer Stelle hatte sich G. für eine Betonung der Differenz als Grundlage der Anerkennung in den christlich-islamischen Be- 
ziehungen ausgesprochen. ${ }^{6}$ Aus pragmatischen Gründen - im Interesse der friedlichen Koexistenz in der Gesellschaft - sei der (kritische) Dialog mit dem Islam wichtig. Dabei müssten Konflikte offen ausgetragen und dürften nicht durch Harmonisierungsstrategien überdeckt werden.

Einer Theologie der Religionen - wie im Grunde jeder Theologie - gegenüber bleibt G. zurückhaltend, weil er sie in der Gefahr stehen sieht, die Prozesse kommunikativer, konfliktorientierter Konsensbildung durch normative Glaubenssätze mit unbedingtem Wahrheitsanspruch zu unterlaufen. Religion ist für ihn die Kompetenz, mit dem Leben »in der grundlegenden Antlitz-Antwort-Differenz der Anderen zu mir in der Spur des fremden Gottes umzugehen« (259). Doch wie ist diese Spur zu identifizieren und zu unterscheiden? Sie kann nicht nur negativ als Relativierung aller Mächte, ethischer Normen und Wahrheitsansprüche bestimmt sein. Um solche Identifizierungen geht es im Dialog und dann auch in der Theologie der Religionen. Wenn darauf verzichtet wird, bleibt das »Ethos der bedingungslosen Verantwortung dem Anderen-Fremden gegenüber« (ebd.) ohne Grund und damit auch ohne Verpflichtungskraft.

Die Zulassung und Wertschätzung von Alterität als Voraussetzung für die Pluralismusfähigkeit einer Religionsform ist auch das Thema der Überlegungen von Eilert Herms zum »Zusammenleben im Widerstreit der Weltanschauungen«. In pluralistischen Gesellschaften treffen nicht nur unterschiedliche Lebens- und Handlungsorientierungen aufeinander, sondern auch die ihnen zugrunde liegenden verschiedenen - oft gegensätzlichen - Menschenund Weltverständnisse. Diese divergierenden und konfligierenden Bezugsrahmen für die Deutung des Lebens und der Lebenswelt können nur dann neben- und miteinander bestehen, wenn sie in sich selbst pluralismusfähig sind, das heißt Andersheit zulassen und anerkennen können. Was aber befähigt sie dazu? Worin bestehen die Bedingungen der Möglichkeit, weltanschauliche und religiöse Vielfalt wertzuschätzen?

Am roten Faden dieser Frage hat H. Vorträge und Aufsätze zu verschiedenen sozialethischen Themen aufgefädelt. Darin gibt er - vom Standort des christlichen Menschenverständnisses aus - zum einen eine grundsätzliche Antwort auf die skizzierte Leitfrage, zum anderen aber setzt er sich mit außerchristlichen Menschen- und Weltbildern auseinander. Diese Standortverpflichtetheit entspricht seiner These: Zusammenleben unterschiedlicher Weltanschauungen ist möglich, wenn und sofern sich diese auf sich selbst

${ }^{6}$ Siehe dazu U. Gerber: Interreligiöser Dialog zur Friedensförderung. Abgrenzung Toleranz - Differenz, in: ders. (Hg.), Auf die Differenz kommt es an - interreligiöser Dialog mit Muslimen, Leipzig 2006, 63-78. 
und auf die Bedingungen ihres Gewordenseins, auf ihre Gebundenheit an Prägungen und Erfahrungen, auf ihre unhintergehbare Perspektivität zurückbesinnen, wenn sie also ein Bewusstsein ihrer Relativität entwickeln und kultivieren. Dazu müssen sie grundlegend zwischen der Wirklichkeit und den Verständnissen der Wirklichkeit unterscheiden. Nicht aus dem Überstieg über die eigene partikulare Weltsicht - hin zu einer vermeintlich weltanschaulich neutralen Vogelperspektive auf die Wirklichkeit -, sondern aus der Vertiefung in die eigene Weltsicht ergeben sich nach $\mathrm{H}$. die Bedingungen für eine friedliche Koexistenz der verschiedenen Weltanschauungen.

In den ersten vier Beiträgen legt H. seine Sicht des christlichen Menschenverständnisses dar. Dabei kommt es ihm nicht nur auf die Entfaltung seiner Inhalte, sondern vor allem auf die Rekonstruktion seiner Konstitution an. Er will zeigen, wie sich dieses Verständnis gebildet hat, wie es sich vom grundlegenden Erschließungsereignis des Christusgeschehens aus in einer Deutegeschichte entwickelt hat und sich immer weiter entwickelt. Diese Selbstaufklärung über die eigene Bildungsgeschichte soll exemplarische Bedeutung auch für die Selbstreflexion anderer Weltsichten und Menschenbilder haben. Im fünften Beitrag, der dem gesamten Band den Titel gegeben hat, ist dieses Programm zusammengefasst.

In den drei folgenden Aufsätzen setzt sich H. mit Religion auseinander, wobei er diesen Begriff im Gefolge Schleiermachers als 》Gewisssein über die universalen Bedingungen des Menschseins, über seinen Ursprung und seine Bestimmung« (p. XIV) auffasst. Unter Verweis auf den Gemeinschaftsbezug religiöser Gewissheitsbildungen lehnt H. die Vorstellung ab, Religion sei Privatsache.

In den weiteren Beiträgen verzweigen sich die Themen in verschiedene Bereiche der Sozialethik: Die Frage nach der gesellschaftlichen Ordnung des menschlichen $\mathrm{Zu}$ sammenlebens wird im Blick auf die Bedeutung von Eliten diskutiert. Eine weitere Gruppe von Texten widmet sich der Anwendung des christlichen Menschenverständnisses auf wirtschafts-, wissenschafts- und technikethische Problemstellungen, wie sie sich etwa aus der Nutzung der Kernenergie, der Präimplantationsdiagnostik und der Stammzellenforschung ergeben. Die drei Beiträge zum Thema Sport fragen nach dem Ethos dieses Lebensbereichs und seinen Begründungen. Überlegungen zu einer dem weltanschaulichen Pluralismus angemessenen Ordnung des Bildungswesens, zum Verhältnis der Geschlechter und zum Zusammenleben in Ehe und Familie schließen den Band ab.

Erhellende Kraft entfalten die unterschiedlichen, sich teilweise überschneidenden Beiträge weniger in den verklausulierten Entfaltungen des christlichen Menschenverständnisses als in seiner Anwendung auf sozialethische Fragen. Wenn auch die sehr geprägte Sprache die Lektüre nicht gerade erleichtert, so findet sich der Leser für seine Mühe doch durch instruktive Analysen - etwa zu wirtschafts- oder medizinethischen Fragen - belohnt. Mit dem Thema des Bandes haben diese Analysen insofern noch zu tun, als sich H.s Erkenntnisinteresse stets auf die Begründungszusammenhänge richtet - auf die weltanschaulichen Fundamente. In der Zurückführung ethi- 
scher auf fundamentalethische, anthropologische Grundlegungen liegt seine Stärke.

Während die Studien von Arens und Gerber von religionssoziologischen und -philosophischen Deutungen der gegenwärtigen Religionstopographie im deutschsprachigen Raum ausgehen, setzt der von Reinhold BerNhardt und Perry Schmidt-Leukel herausgegebene Band »Multiple religiöse Identität. Aus verschiedenen religiösen Traditionen schöpfen« religionspsychologisch bei den individualreligiösen Identitätsbildungsprozessen an. Eine von der »Identity Foundation« in Auftrag gegebene Studie der Universität Hohenheim kam im Jahr 2006 zu dem Ergebnis, dass die »Traditions-Christen« immer mehr einer Gruppe der »Religiös Kreativen« weichen, die ihr religiöses Selbstverständnis aus Fragmenten der Religionstraditionen und eigener Reflexion speisen. ${ }^{7}$ Es ist dabei durchaus nicht nur an die unverbindlichen postmodernen Religionsbricolagen zu denken, die unter dem Begriff der »Patchwork-Religiosität« diskutiert werden, die Gerber im Typus der Erleichterungsreligion kategorisiert und Arens als religiösen Individualismus beschrieben hatte, sondern auch an das ernsthafte Bemühen von Menschen, ihr spirituelles Lebenswasser aus mehreren Brunnen zu beziehen. Es können dies Menschen sein, die im Laufe ihrer Persönlichkeitsentwicklung als sspirituelle Wandererı durch eigene Entscheidung einen solchen Weg einschlagen oder auch solche, die in zwei oder mehrere Religionstraditionen hinein sozialisiert worden sind und in beiden leben wollen; etwa Kinder von Eltern, die unterschiedlichen Religionen angehören und sich in beiden Traditionen beheimaten wollen, oder Migranten, die in eine fremde religiöse Umgebung gekommen sind, sich auch religiös für diese Umgebung öffnen, dabei aber in ihrer religiösen Herkunftsidentität verwurzelt bleiben wollen. Ulrich Schoen spricht von »Brückenmenschen ${ }^{8}$, die zwei oder mehreren Sprachen, Kulturen und Religionen angehören und im Alltag ihres Lebens zwischen ihnen Vermittlungen vornehmen.

Für das Phänomen der religiösen Mehrfachzugehörigkeit, bei dem sich eine Person zwei oder mehreren religiösen Traditionen zugleich zugehörig fühlt, sich zu ihnen bekennt und sie praktiziert, hat sich in der englischsprachigen religionswissenschaftlichen und religionstheologischen Forschung der Begriff »multiple religious belonging« eingebürgert. Nicht ganz bedeutungsgleich damit ist der Begriff »multiple religious identity«, der den Aspekt der

${ }^{7}$ http://www.identityfoundation.de/fileadmin/templates_identityfoundation/downloads/presse/frauen_spiritualitaet/PM_Lang_Studie_Spiritualitaet.pdf.

U. Schoen, Mensch sein in zwei Welten. Bi-Identität in Sprache, Religion und Recht (Ökumenische Studien 11), Münster 2000, 47 ff. 170 ff. 211 ff. 
Identitätsformation in den Vordergrund stellt. »Multiple religious belonging« betont dagegen mehr das Moment der Zugehörigkeit, Partizipation, Bindung und Verpflichtung gegenüber einer religiösen Tradition und ihrer Trägergemeinschaft. Sachgemäß erscheint auch der Begriff »multiple participations«. In der deutschsprachigen Diskussion spricht man von »religiöser Mehrfachzugehörigkeit«, von »hybrider Religiosität« oder »multipler religiöser Identität« oder in Analogie zur Sprache von »religiöser Mehrsprachigkeit«.

Als die koreanische Theologin Chung Hyun Kyung im Rahmen ihres inszenierten Eröffnungsvortrags bei der VII. Vollversammlung des ÖRK in Canberra 1991 nach koreanisch-schamanischer Tradition Han-Geister als Mittler des heiligen Geistes anrief, entbrannte ein Sturm der Entrüstung. Eine intensive Auseinandersetzung über die Inkulturation des Evangeliums und die in Ostasien verbreitet anzutreffende Verbindung christlicher Spiritualität mit den dort beheimateten religiösen Traditionen schloss sich an. 1993 erklärte Frau Chung auf einer Konferenz: »Es kommt mir so vor, als sei ich im Bauch Schamanin, im Herzen Buddhistin, in der rechten Gehirnhälfte Konfuzianerin und in der linken Gehirnhälfte Christin. «9 Solchen religiösen Selbstverständnissen widmet sich der Band.

Nach dem systematisierenden Überblick, den Catherine Cornille vom Boston College über die Thematik gibt, werden in exemplarischen Einzelstudien drei Persönlichkeiten vorgestellt, die je auf ihre eigene Weise aus mehreren religiösen Traditionen schöpfen: Henri Le Saux, der in Indien unter dem Namen Swami Abhishiktananda lebte (Christian Hackbarth-Johnson), Raimon Panikkar, der seinen Lebensweg in den bekannten Worten zusammengefasst hat: »Ich bin als Christ igegangen<, ich habe mich als Hindu sgefunden und ich `kehre` als Buddhist szurückı, ohne doch aufgehört zu haben, ein Christ zzu sein « ${ }^{10}$ (Bernhard Nitsche) und Frithjof Schuon, der in einen Sufiorden eintrat, bei der Lektüre der Bhagavadgita ein Bekehrungserlebnis hatte und daraufhin tiefer in den mystischen Islam vordrang, ohne seine christlichen Wurzeln zu verlassen (André Gerth).

Im zweiten Teil des Bandes wird das Phänomen der religiösen Mehrfachzugehörigkeit aus unterschiedlichen religionswissenschaftlichen Perspektiven beleuchtet. Joachim Gentz blickt nach China, wo die drei Lehren des Konfuzianismus, Daoismus und Buddhismus durch den Harmoniegedanken verbunden wurden und werden. Christoph Bochinger gibt einen Einblick in den Individualsynkretismus der sspirituellen Wanderers im Westen und stellt exemplarisch eine Studie zu spirituellen Orientierungen evangelischer und katholischer Christen in Franken vor. Aus religionspsycho-

9 Zitiert in: Donna F. G. Hailson, Church Women Unorthodox. The Theological Corruption of Church Women United. In: Touchstone, Juli/August 1998 (http:// www.touchstonemag.com/archives/article.php?id=11-04-044-f). Siehe auch: Chung Hyun Kyung, Schamanin im Bauch, Christin im Kopf, Stuttgart 1992.

10 R. Panikkar, Der neue religiöse Weg. Im Dialog der Religionen leben, München 1990, 51 . 
logischer Sicht geht Tatjana Schell auf das Phänomen der multiplen religiösen Identitätsformationen ein und ordnet sie in den Kontext der Theorien der Religiositätsentwicklung ein.

Im dritten Teil werden religionstheologische Aspekte erörtert. Karl Baier fragt nach dem Integrationszentrum bei der Verbindung unterschiedlicher religiöser Traditionen und Spiritualitätsformen. Dabei geht er vom Begriff des habitus, der dauerhaften ganzheitlichem leib-seelischen Gestimmtheit aus. »Eine Mehrfachzugehörigkeit ist spirituell möglich, wenn das Habitussystem, das in einer Tradition eingeübt und gelebt wird, mit dem der anderen Tradition kompatibel ist, also die charakteristischen Haltungen zur Welt und zur letzten Wirklichkeit nicht im Widerspruch zueinander stehen und einander bereichern können« (213). Von Erfahrungen mit einer zunehmenden Flexibilisierung religiöser Identitätskonzepte in den Niederlanden berichtet Manuela Kalsky in ihren Überlegungen zur religiösen Mehrfachidentität. Der Frage nach einer Bewertung solcher Identitätsbildungen stellt sich Perry Schmidt-Leukel vom Standpunkt der Pluralistischen Religionstheologie aus. Den Vorwurf des »Synkretismus« erörtert Reinhold Bernhardt. Michael von Brück schließt den Band mit seinen Überlegungen zu einer Theologie multipler religiöser Identität ab.

Im Durchgang durch die Beiträge zeigt sich, dass es sich bei der skizzierten religiösen Mehrsprachigkeit keineswegs um eine Erscheinung handelt, die sich lediglich bei einzelnen religiösen Virtuosen findet. Auf mehr oder weniger reflektiertem Niveau begegnet sie in der Formation des religiösen Selbstverständnisses vieler Zeitgenossen auch in Mitteleuropa. Nicht nur die Praktische Theologie, sondern auch die Systematische Theologie wird sich dieser Entwicklung zu stellen haben. Ein polemisch-aufgeladener Synkretismusbegriff ist dabei wenig hilfreich.

Ein weiterer, kürzlich erschienener Band aus der Reihe »Beiträge zu einer Theologie der Religionen«, herausgegeben von Reinhold Bernhardt und KLaus von Stosch, stellt einen neuen Ansatz in der religionstheologischen Debatte vor, der in Nordamerika - vor allem am Boston College, an der Boston University und an der Harvard Divinity School - entwickelt worden ist und im deutschsprachigen Raum zunehmend auf Interesse stößt: die »Komparative Theologie«. Er ist nicht zuletzt aus einer Kritik an der bisherigen, am Dreierschema von Exklusivismus, Inklusivismus und Pluralismus orientierten religionstheologischen Diskussion hervorgegangen und versteht sich als Alternative dazu. Die Beziehung zwischen dem Christentum und anderen Religionen soll dabei nicht durch solche formale Raster bestimmt werden, sondern durch den mikrologischen Vergleich einzelner Vorstellungsund Praxisformen. Diese sollen stiefengrammatisch auf ihre Intention und ihre Funktion im System der jeweiligen Religionstradition hin befragt und dann zueinander in Beziehung gesetzt werden. Dies geschieht aus der Perspektive der christlichen Theologie, nicht also im Sinne einer vergleichenden Religionswissenschaft. 
In diesem Band wird der Forschungsansatz vorgestellt und in Vergleichen zwischen christlichen, muslimischen und buddhistischen Traditionselementen erprobt. Es zeigt sich dabei, dass die Komparative Theologie keine homogene Schulbildung ist, sondern ein in sich vielfältiges Experimentierfeld, auf dem die einzelnen Vertreter von verschiedenen philosophischen Grundlagen aus operieren, unterschiedliche methodische Wege gehen und in der Zielrichtung eigene Akzente setzen.

Der Band präsentiert im ersten Teil programmatische Perspektiven. Klaus von Stosch, Robert Cummings Neville und Keith Ward stellen das Projekt der »Komparativen Theologie» vor, Ulrich Winkler kommentiert und diskutiert die Zugänge von Ward und Neville, Norbert Hintersteiner gibt Hinweise zur Entstehung dieser Bewegung und nimmt eine Ortsbestimmung vor.

Die Beiträge des zweiten und dritten Teils nehmen exemplarische interreligiöse Vergleiche vor. Auch wenn nicht alle der Autoren der Komparativen Theologie im engeren Sinne zuzurechnen sind, so zeigt sich in ihren Studien doch, wie erhellend interreligiöse Vergleiche zum Verständnis spezifisch christlicher Glaubensüberlieferungen sind. Im zweiten Teil, in dem Vergleiche zwischen christlichen und islamischen topoi angestellt werden, beleuchtet Pim Valkenberg das Offenbarungsverständnis des Islam. Christine Niggemann und Klaus von Stosch arbeiten dessen ästhetische Dimension heraus. Anja Middelbeck-Varwick vergleicht das Ringen mit der Theodizeefrage in beiden Traditionen. Im dritten Teil - christlich-buddhistische Vergleiche setzt James Fredericks Lehren des mittelalterlichen, japanisch-buddhistischen Denkers Dōgen Kigen zum ursprünglichen Personsein und zur Buddha-Natur in Beziehung züm Personbegriff in der christlichen Trinitätstheologie. Ebenfalls an Dōgen knüpft Jürgen Mohn in seiner vergleichenden Untersuchung zum Zeitverständnis in Buddhismus und Christentum an, wobei er sich hier besonders auf Augustin bezieht. Dabei arbeitet er den Unterschied zwischen einer religionswissenschaftlichen Komparatistik und der Komparativen Theologie heraus. Auch Michael Hüttenhoff setzt sich mit dem Personbegriff in Christentum und Buddhismus auseinander, wobei er allerdings die Anthropologie als Bezugsrahmen nimmt. Die buddhistische Problematisierung des Selbst stelle nicht - wie oft angenommen - das Personsein des Menschen, sondern vor allem die Lehre von der Unsterblichkeit der Seele in Frage.

Christiane Tietz resümiert und kommentiert abschließend die Beiträge dieses Bandes und stellt dabei die Konzepte der Komparativen Theologie im Überblick noch einmal zur Diskussionen. Sie fragt nach deren Prämissen, Methoden und Zielen und fordert weitere Klärungen ein.

Das Projekt der Komparativen Theologie wird im deutschsprachigen Raum vor allem von Ulrich Winkler (Salzburg) ${ }^{11}$ und von Klaus von Stosch (Paderborn) $)^{12}$ betrieben. Sie sehen darin einen nicht nur für das interreligiöse

11 Siehe dazu das Themenheft der SaThZ 11, 2/2007.

12 Vor kurzem wurde dort das »Zentrum für Komparative Theologie und Kulturwissenschaften (ZeKK) « gegründet. Von Stosch hat im Verlag Ferdinand Schöningh eine Publikationsreihe unter dem Titel »Beiträge zur Komparativen Theologie« lan- 
Verstehen, sondern auch für die Systematische Theologie ertragreichen Weg. Denn in der Auseinandersetzung mit vergleichbaren Lehr- und Praxisformen anderer religiöser Traditionen lassen sich wertvolle Einsichten für die Entfaltung theologischer Inhalte gewinnen.

ciert, deren erster Band im Frühjahr 2010 unter dem Titel »erwundete Gewissheit. Strategien im Umgang mit Verunsicherung in Islam und Christentum« (hg. von Jürgen Werbick. Muhammad Sven Kalisch und Klaus von Stosch), erschienen ist. 